\title{
Pairing phenomena and superfluidity of atomic Fermi gases in a two-dimensional optical lattice: Unusual effects of lattice-continuum mixing
}

\author{
Lin Sun, ${ }^{1}$ Jibiao Wang, ${ }^{2}$ Xiang Chu, ${ }^{1}$ and Qijin $\mathrm{Chen}^{3,4,5,1, \text {, }}$ \\ ${ }^{1}$ Department of Physics and Zhejiang Institute of Modern Physics, \\ Zhejiang University, Hangzhou, Zhejiang 310027, China \\ ${ }^{2}$ School of Materials and New Energy, South China Normal University, Shanwei, Guangdong 516625, China \\ ${ }^{3}$ Hefei National Laboratory for Physical Sciences at Microscale and School of Physics, \\ University of Science and Technology of China, Hefei, Anhui 230026, China \\ ${ }^{4}$ Shanghai Branch, CAS Center for Excellence and Synergetic Innovation Center in Quantum Information and Quantum Physics, \\ University of Science and Technology of China, Shanghai 201315, China \\ ${ }^{5}$ Shanghai Research Center for Quantum Sciences, Shanghai 201315, China
}

(Dated: March 1, 2022)

\begin{abstract}
We study the superfluid behavior of ultracold atomic Fermi gases with a short range attractive interaction in a two-dimensional optical lattice (2DOL) using a pairing fluctuation theory, within the context of BCS-BEC crossover. We find that the mixing of lattice and continuum dimensions leads to exotic phenomena. For relatively large lattice constant $d$ and small hopping integral $t$, the superfluid transition temperature $T_{c}$ exhibits a remarkable reentrant behavior as a function of the interaction strength, and leads to a pair density wave ground state, where $T_{c}$ vanishes, for a range of intermediate coupling strength. In the unitary and BCS regimes, the nature of the in-plane and overall pairing changes from particle-like to hole-like, with an unexpected nonmonotonic dependence of the chemical potential on the pairing strength. The BEC asymptotic behaviors exhibit distinct power law dependencies on the interaction strength compared to cases of pure 3D lattice, 3D continuum, and 1DOL. These predictions can be tested in future experiments.

Ann Phys (Berlin) 2022, 2100511
\end{abstract}

\section{INTRODUCTION}

Ultracold Fermi gases have become a powerful platform for investigating condensed matter physics over the past decade, due to their multiple experimentally tunable control parameters [1, 2]. They can exhibit a perfect crossover from a BCS type of superfluidity to Bose-Einstein condensation (BEC) of atom pairs, as the pairing strength is tuned from weak to strong via a Feshbach resonance [3]. In particular, they can be put in an optical lattice with a tunable geometry so that rich physics may emerge [4-6]. In a three dimensional (3D) lattice, it is known that the superfluid transition temperature $T_{c}$ scales as $-t^{2} / U$ in the BEC regime, due to "virtual ionization" in the pair hopping process [7, 8], where $U<0$ is the onsite attractive interaction, and $t$ is the hopping integral. This is to be contrasted with the $3 \mathrm{D}$ continuum case, in which $T_{c}$ approaches asymptotically $0.218 T_{F}$ in the BEC regime, with $T_{F}$ being the Fermi temperature. Given the sharp contrast between the 3D continuum and the 3D lattice cases, usual behaviors may be expected when the spatial dimensions contain both lattice and continuum. Recently, as such a latticecontinuum mixed system, the superfluid behavior of Fermi gases in a 1D optical lattice (1DOL), in the context of BCSBEC crossover, has been studied. Interesting effects of latticecontinuum mixing have indeed been found. For example, in the population balanced case, the behavior of $T_{c}$ exhibits lattice effect; $T_{c}$ decreases with increasing coupling strength in the BEC regime, with a scaling of $T_{c}=\pi a n / 2 m$, where $a$ is the $s$-wave scattering length, $n$ the fermion density and $m$ the fermion mass [9]. In contrast, in the presence of population

\footnotetext{
* Corresponding author: qchen@uchicago.edu
}

imbalance [10], $T_{c}$ often approaches a constant BEC asymptote, mimicking its counterpart in the 3D continuum.

Two dimensional optical lattices (2DOL) have also been studied theoretically [11-13]. They corresponds to a 2D array (2D lattice in the $x y$ plane) of $1 \mathrm{D}$ tubes (1D continuum alone the $z$ direction) in experiment, with lattice constant $d$ and hopping integral $t$, subject to a short-range pairing interaction $U(<0)$. 2DOL of ${ }^{6} \mathrm{Li}$ in the (quasi-) $1 \mathrm{D}$ limit has been realized experimentally [14-16]. None of these theoretical and experimental studies paid attention to or was aware of possible lattice-continuum mixing effects in a more general parameter range. Most of them have focused on the 1D or quasi1D physics, including the Fulde-Ferrell-Larkin-Ovchinnikov states (FFLO) state [17] in the presence of a population imbalance. Dimensional crossover was also studied [16] in a population imbalanced Fermi gas of ${ }^{6} \mathrm{Li}$ atoms, with a focus on how phase separation evolves with $t$ and the interactions. Apparently, the lattice-continuum effects may not show up for any arbitrary choices of $d$ and $t$ as well as interaction strength.

In this paper, we investigate the superfluid behavior of a balanced two-component Fermi gas in a 2DOL. We find a much stronger effect of lattice-continuum mixing than in the 1DOL case, besides the wide spread pseudogap phenomena [1]. In particular, two lattice dimensions provide a tighter confinement in the momentum space. Via increasing lattice constant $d$ and decreasing hopping integral $t$, such that the Fermi surface becomes open (in contrast to a 3D closed ellipsoid), the effective number density in the lattice dimensions can go above half filling, and may turn the in-plane and overall pairing from particle-like into hole-like in the BCS and unitary regimes, with chemical potential $\mu$ increasing with increasing pairing strength, while it remains particlelike in the BEC regime. While the decrease of $T_{c}$ in the BEC regime manifests strong lattice effects, we find that for large 
$d$ and small $t, T_{c}$ exhibits a re-entrant behavior in the regime of intermediate pairing strength; a pair density wave (PDW) emerges as the ground state where $T_{c}$ vanishes. In the BEC regime, the momentum space becomes quasi-1D, so that $T_{c}$ approaches $\frac{\sqrt{6} n t d^{4}}{m}|U|^{-1}$ asymptotically, while $\mu \sim-U^{2}$ is dominated by a quadratic dependence on $U$, with the pairing gap $\Delta \sim|\mu|^{3 / 4} \sim|U|^{3 / 2}$. These behaviors are distinct from their counterpart in 3D lattice, 3D continuum or 1DOL.

It is known that the superfluid phase in Fermi gases in 3D optical lattices has yet not been accessible experimentally, due to the difficulty of deep cooling. On the contrary, superfluidity in 1D or 2D optical lattices have been realized long ago, e.g., in the early phase separation observation [18].

\section{THEORETICAL FORMALISM}

\section{A. Pairing fluctuation theory}

To focus on the effects of lattice-continuum mixing, we shall focus on the 2DOL without the complication of population imbalance. Under the one-band tight-binding approximation for the lattice dimensions in the $x y$ plane, the dispersion of noninteracting atoms is given by $\xi_{\mathbf{k}}=\epsilon_{\mathbf{k}}-\mu \equiv$ $k_{z}{ }^{2} / 2 m+2 t\left[2-\cos \left(k_{x} d\right)-\cos \left(k_{y} d\right)\right]-\mu$. Following our recent studies [9, 10, 19, 20], we take $t$ to be small in our calculation, under the constraint $2 m t d^{2}<1$, so that it is physically accessible. The critical coupling strength, which corresponds to a diverging $s$-wave scattering length $a$ in the unitary limit in the presence of the lattice, is given by $U_{c}=-1 / \sum_{\mathbf{k}} 1 / 2 \epsilon_{\mathbf{k}}=-0.16072 \sqrt{2 m} / \sqrt{t} d^{2}$. Note that a big difference between $2 \mathrm{DOL}$ and $1 \mathrm{DOL}$ as well as 3D continuum is that $U_{c}=0$ in the contact potential limit for the latter two cases. As a consequence, $1 / k_{F} a$ as defined via the Lippman-Schwinger equation is upper bounded in the present case. Thus 2DOL is closer to the 3D lattice case in this sense. In comparison with the pure 3D lattice case, however, the momentum distribution will spread to large $k_{z}$ (in the BEC case or for small $t$ and large $d$ ), leading to a quasi-1D momentum space, with the kinetic energy distributed mainly along the $k_{z}$ direction. For 3D lattice, which has a finite momentum space defined by the first Brillouin zone (BZ), there is a finite filling factor for a given fermion density. However, the overall filling factor is zero for 2DOL. Nevertheless, as we show below, one can tune $t$ and $d$ such that the effective filling factor within the two lattice dimensions varies between 0 and 1 .

With the modified atomic dispersion $\xi_{\mathrm{k}}$ for 2DOL, we follow the pairing fluctuation theory previously developed for the pseudogap physics in the cuprates [21], which has been extended to address the BCS-BEC crossover in ultracold atomic Fermi gases [1]. This theory goes beyond the BCS meanfield approximation by self-consistently including finite momentum pairing correlations in the single particle self energy. Thus the self energy $\Sigma(K)=\Sigma_{s c}(K)+\Sigma_{p g}(K)$ contains two parts, where the Cooper pair condensate contribution $\Sigma_{s c}(K)=-\Delta_{s c}^{2} G_{0}(-K)$ vanishes above $T_{c}$, and finite momentum pair contribution $\Sigma_{p g}(K)=\sum_{Q} t_{p g}(Q) G_{0}(Q-K)$ exists both above and below $T_{c}$. Here $G_{0}$ and $G$ are the non-interacting and full Green's functions, respectively, $\Delta_{s c}$ the order parameter and $t_{p g}(Q)=U /[1+U \chi(Q)]$ the pairing $T$ matrix, with pair susceptibility $\chi(Q)=\sum_{K} G_{0}(Q-$ $K) G(K)$. We take $\hbar=k_{B}=1$ and use four momenta $K \equiv\left(\omega_{n}, \mathbf{k}\right), Q \equiv\left(\Omega_{l}, \mathbf{q}\right), \sum_{Q} \equiv T \sum_{l} \sum_{\mathbf{q}}$, etc, where $\omega_{n}\left(\Omega_{l}\right)$ is the odd (even) Matsubara frequency, following the notations of Ref. [21] [22].

We now recapitulate the main result of this theory. In the superfluid phase, one can define the pseudogap via $\Delta_{p g}^{2}=$ $-\sum_{Q} t_{p g}(Q)$, so that the total gap $\Delta$ is given by $\Delta^{2}=\Delta_{s c}^{2}+$ $\Delta_{p g}^{2}$, which leads to the self energy $\Sigma(K) \approx-\Delta^{2} G_{0}(-K)$, and the BCS form of the full Green's function $G(K)=$ $\frac{u_{\mathbf{k}}^{2}}{i \omega_{n}-E_{\mathbf{k}}}+\frac{v_{\mathbf{k}}^{2}}{i \omega_{n}+E_{\mathbf{k}}}$, where $u_{\mathbf{k}}^{2}=\left(1+\xi_{\mathbf{k}} / E_{\mathbf{k}}\right) / 2, v_{\mathbf{k}}^{2}=$ $\left(1-\xi_{\mathbf{k}} / E_{\mathbf{k}}\right) / 2$, and $E_{\mathbf{k}}=\sqrt{\xi_{\mathbf{k}}^{2}+\Delta^{2}}$. Then we have the number equation,

$$
n=\sum_{\mathbf{k}}\left[\left(1-\frac{\xi_{\mathbf{k}}}{E_{\mathbf{k}}}\right)+2 \frac{\xi_{\mathbf{k}}}{E_{\mathbf{k}}} f\left(E_{\mathbf{k}}\right)\right],
$$

where $f(x)$ is the Fermi distribution function. At $T \leq T_{c}$, the Thouless criteria for pairing instability, $U^{-1}+\chi(0)=0$, leads to the gap equation,

$$
0=\frac{1}{U}+\sum_{\mathbf{k}}\left[\frac{1-2 f\left(E_{\mathbf{k}}\right)}{2 E_{\mathbf{k}}}\right]
$$

To evaluate the pseudogap, one can Taylor expand $t_{p g}^{-1}(Q)$ on the real frequency axis, after analytical continuation, $t_{p g}^{-1}(\Omega, \mathbf{q}) \approx a_{1} \Omega^{2}+a_{0}\left(\Omega-\Omega_{\mathbf{q}}+\mu_{p}\right)$, with $\Omega_{\mathbf{q}}=B q_{z}^{2}+$ $2 t_{B}\left[2-\cos \left(q_{x} d\right)-\cos \left(q_{y} d\right)\right]$, and $\mu_{p}=0$ at $T \leq T_{c}$. Here $B=1 / 2 M$, with $M$ being the effective pair mass in the $z$ direction, and $t_{B}$ the effective pair hopping integral in the $x y$ plane. We stress that the $a_{1}$ term serves as a small quantitative correction in the BEC regime, where $a_{1} T_{c} \ll a_{0}$. Then this $T$-matrix expansion leads to the pseudogap equation

$$
\left|a_{0}\right| \Delta_{p g}^{2}=\sum_{\mathbf{q}} \frac{b\left(\tilde{\Omega}_{\mathbf{q}}\right)}{\sqrt{1+4 \frac{a_{1}}{a_{0}} \Omega_{\mathbf{q}}}},
$$

where $b(x)$ is the Bose distribution function and $\tilde{\Omega}_{\mathbf{q}}=$ $\left[\sqrt{a_{0}^{2}+4 a_{1} a_{0} \Omega_{\mathbf{q}}}-a_{0}\right] / 2 a_{1}$ is the pair dispersion, which reduces to $\tilde{\Omega}_{\mathbf{q}}=\Omega_{\mathbf{q}}$ when $a_{1} T_{c} / a_{0} \ll 1$, e.g., in the BEC regime. In this case, one can see that the pair density is roughly given by $n_{p}=a_{0} \Delta^{2}$

Equations (1)-(3) form a closed set of self-consistent equations, which can be used to solve for $\left(\mu, \Delta_{p g}, T_{c}\right)$ with $\Delta_{s c}=$ 0 . For our numerics, we define Fermi momentum $k_{F}=$ $\left(3 \pi^{2} n\right)^{1 / 3}$ and Fermi energy $E_{F} \equiv k_{B} T_{F}=\hbar^{2} k_{F}^{2} / 2 m$, as for the $3 \mathrm{D}$ continuum with the same density $n$ and atomic mass $m$.

\section{B. Asymptotic behaviors in the BEC limit}

The asymptotic behavior at $T_{c}$ in the BEC limit can be solved analytically, with $\mu \rightarrow-\infty$, so that $f\left(E_{\mathbf{k}}\right)=f\left(\xi_{\mathbf{k}}\right)=$ 
0 . From the gap equation, we obtain

$$
\mu=-\frac{m}{8 d^{4}} U^{2}+\frac{3 n}{4}|U| .
$$

The leading order quadratic dependence of $\mu$ on $U$ is related to the quasi-one dimensionality caused by the restricted momentum space for $\left(k_{x}, k_{y}\right)$ within the first BZ. This should be contrasted to the 3D lattice case, for which $\mu \sim U$ in the BEC limit [23]. From the number equation, we get

$$
\Delta=\left(\frac{8 d^{2} n}{\sqrt{2 m}}\right)^{1 / 2}|\mu|^{3 / 4} .
$$

This $\Delta \sim|\mu|^{3 / 4} \sim|U|^{3 / 2}$ scaling behavior should be contrasted to $\Delta \sim \mu \sim|U|$ for 3D lattice, $\Delta \sim|\mu|^{1 / 2}$ for 1DOL (quasi-2D system) [9], and $\Delta \sim|\mu|^{1 / 4}$ for 3D continuum [1].

For the effective pair mass in the continuum dimension and the effective pair hopping integral in the lattice dimensions, one can easily obtain

$$
B=\frac{1}{4 m}, \quad \text { and } \quad t_{B}=\frac{3 t^{2}}{4|\mu|},
$$

which yields

$$
n \approx \frac{\sqrt{T_{c}}}{\sqrt{\pi B} d^{2}} L i_{1 / 2}\left(e^{-4 t_{B} / T_{c}}\right)
$$

in the BEC regime via the pseudogap equation (3), where $L i_{s}(z)=\sum_{n=1}^{\infty}\left(z^{n} / n^{s}\right)$ is the polylogarithm function. Note here the $a_{1}$ term is negligible, so that $n_{p}=a_{0} \Delta^{2}=n / 2$. Thus we obtain the BEC asymptotic solution

$$
T_{c} \approx 2 n d^{2} \sqrt{B t_{B}} \approx \frac{\sqrt{6} n t d^{4}}{m}|U|^{-1}
$$

using the lowest order expansion, $L i_{1 / 2}^{-2}(1-z) \approx z / \pi$ for $z \rightarrow 0$. This indicates that $T_{c}$ scales inversely proportional to $U$ in the deep BEC regime, similar to the 3D lattice case.

\section{RESULTS AND DISCUSSIONS}

Shown in the inset of Fig. 11(a) is a typical phase diagram of Fermi gases in 2DOL, with $t / E_{F}=0.05$ and $k_{F} d=2$, which satisfies $2 m t d^{2}=0.2<1$. A semi-log scale is used to show the full interaction range. The black solid and red dashed curves are $T_{c}$ and the pair formation temperature $T^{*}$ (which is approximated by the mean-field solution for $T_{c}$ ), respectively, so that the yellow shaded area indicates the superfluid phase. A pseudogap state exists between $T^{*}$ and $T_{c}$. As usual, $T_{c}$ follows the BCS solution in the weak-coupling regime. Then a pseudogap emerges as $|U|$ increases, which suppresses $T_{c}$ relative to $T^{*}$. After reaching a maximum around unitarity at $U=U_{c}, T_{c}$ starts to decrease due to a shrinking Fermi surface. A minimum is reached when $\mu=0$, where the Fermi surface shrinks to zero and essentially all fermions have paired up. Beyond this point, as the pairing becomes stronger,
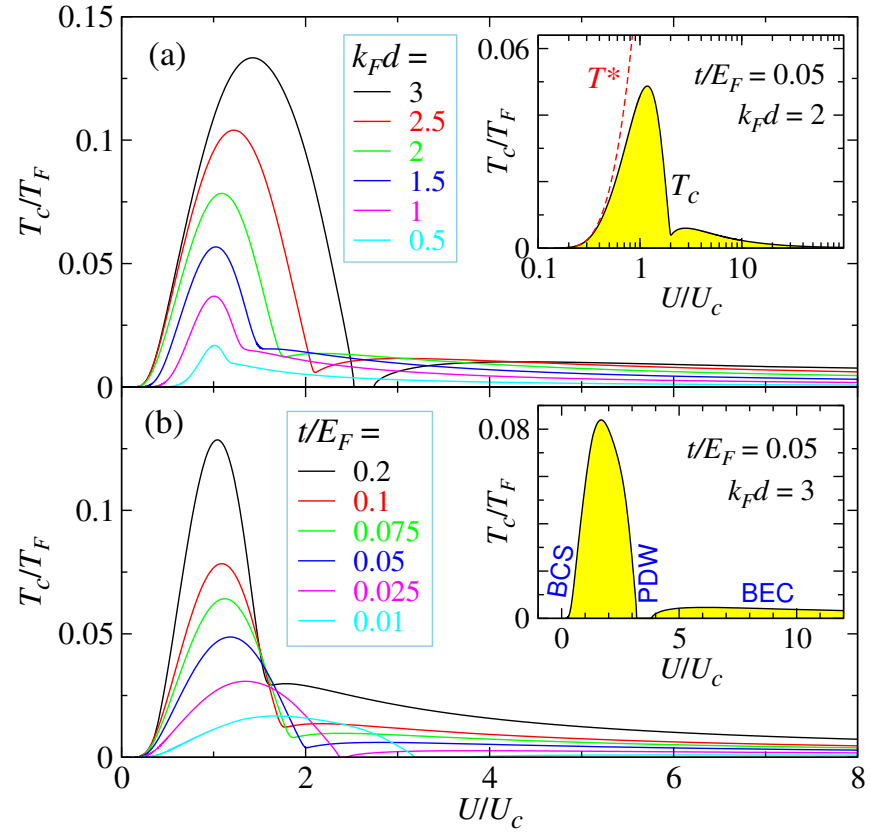

Figure 1. Phase diagram and effect of varying $d$ and $t$ : Behavior of $T_{c}$ versus $U / U_{c}$ with (a) fixed $t / E_{F}=0.1$ for varying $k_{F} d$, and (b) fixed $k_{F} d=2$ for varying $t / E_{F}$, as labeled. The insets of (a) and (b) show typical phase diagrams without and with a PDW phase, respectively.

the system enters the bosonic regime, with $\mu<0$, and $T_{c}$ first rises due to shrinking pair size and hence decreasing pair mass, and then decreases towards the BEC asymptote, as governed by the lattice effect [7, 8].

We note that a constant Hartree energy, $\Sigma_{\mathrm{HF}}=U n / 2$, has been absorbed in our chemical potential $\mu$ [24]. The actual chemical potential is given by $\mu^{\prime}=\mu+U n / 2$. Nevertheless, we emphasize that the vanishing of Fermi surface is defined by $\mu=0$ rather than $\mu^{\prime}=0$. [25]

To demonstrate the effect of varying $d$ and $t$, we plot in Fig. 1 $T_{c}$ versus $U / U_{c}$ with (a) fixed $t / E_{F}=0.1$ but for varying $k_{F} d$ from 0.5 through 3 , and (b) fixed $k_{F} d=2$ but for varying $t / E_{F}$ from 0.01 through 0.2 . For fixed $t$ in panel (a), the maximum $T_{c}, T_{c}{ }^{\max }$, increases with $d$. At the same time, the entire $T_{c}$ curve also expands horizontally, as $d$ increases. Since the lattice momentum $\left(k_{x}, k_{y}\right)$ is restricted to the first BZ, fermions almost fully occupy the $x y$-plane states when $t$ is small [9]. As $d$ increases, the BZ shrinks, and more fermions have to occupy higher $k_{z}$ state, so that $\mu$ is pushed up, leading to higher $T_{c}$. Similarly in panel (b), as $t$ decreases with fixed $d, T_{c}{ }^{\text {max }}$ decreases and moves toward the BEC side of unitarity, as if the entire $T_{c}$ curve is expanding horizontally toward the BEC regime. For fixed $d$, more fermions will occupy the first BZ in the $x y$ plane, as $t$ decreases. This will remove the high $k_{z}$ fermions, and decrease $\mu$. Meanwhile, a small $t$ causes difficulty for pair hopping and thus suppresses $T_{c}$, making the system more 1D.

Remarkably, we can see that $T_{c}$ exhibits a reentrant behavior for large $d$ and small $t$. This is illustrated more clearly as an example in the inset of Fig. 1(b) for $k_{F} d=3$ and 


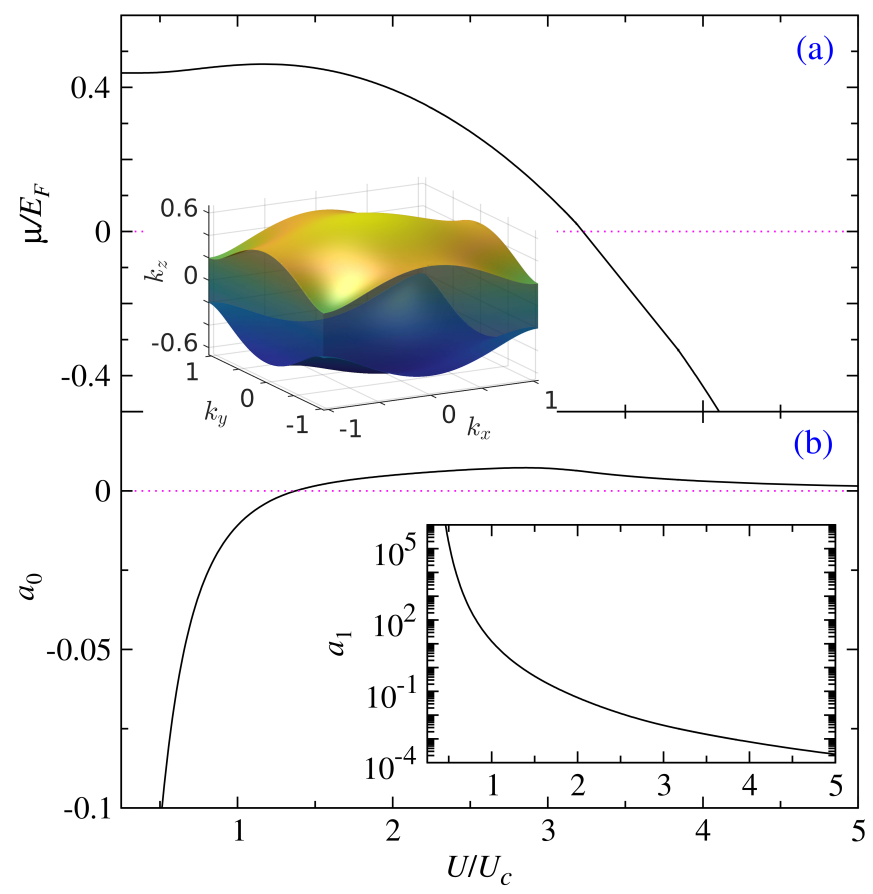

Figure 2. Evolution of (a) $\mu$ and (b) the coefficients $a_{0}$ and $a_{1}$ (inset) at $T_{c}$ as a function of $U / U_{c}$ for $t / E_{F}=0.05$ and $k_{F} d=3$. $T=0$ is used in the PDW state. Also shown is the 3D plot of the corresponding Fermi surface.

$t / E_{F}=0.05$. For a range of intermediate coupling strength, $T_{c}$ shuts off completely before it recovers at stronger couplings in the BEC regime, and PDW states emerge where $T_{c}$ vanishes, which is also found in dipolar Fermi gases within our pairing fluctuation theory [26].

Shown in Fig. 2 are the behaviors of (a) $\mu$ and (b) the coefficients $a_{0}$ and $a_{1}$ (inset) as a function of $U / U_{c}$ for $t / E_{F}=$ 0.05 and $k_{F} d=3$. It is remarkable that $\mu$ increases first as the pairing becomes stronger, which is distinct from the 3D continuum and 3D lattice cases below half filling. Even more remarkable is that $a_{0}<0$ before $\mu$ reaches its maximum near $U / U_{c}=1.38$, even though the monotonic behavior of $a_{1}$ is rather conventional. For larger $U / U_{c}, a_{0}$ becomes positive, and then starts to decrease with $U / U_{c}$ in the BEC regime, where $\mu$ becomes negative. The unusual behaviors of $\mu$ and $a_{0}$ can be explained by the Fermi surface, as shown in the inset. It has open ends, in contrast to a closed ellipsoid for cases of large $t$ and small $d$. As is well known, in the 3D lattice case above half filling, the Fermi surface becomes hole-like, for which $a_{0}$ is negative and the chemical potential $\mu$ increases as pairing strength increases. This can be easily understood via a particle-hole transformation. Therefore, the sign of $a_{0}$ determines whether the fermion pairs are particlelike or hole-like. The negative sign has to do with the negative quasiparticle velocity on the hole-like Fermi surface. For the present 2DOL case shown in Fig. 2, the effective filling factor in the first BZ of the $x y$ plane is above $1 / 2$, so that the contributions from lattice dimensions dominate the sign of $a_{0}$ in the unitary and weak-coupling regimes, even though the

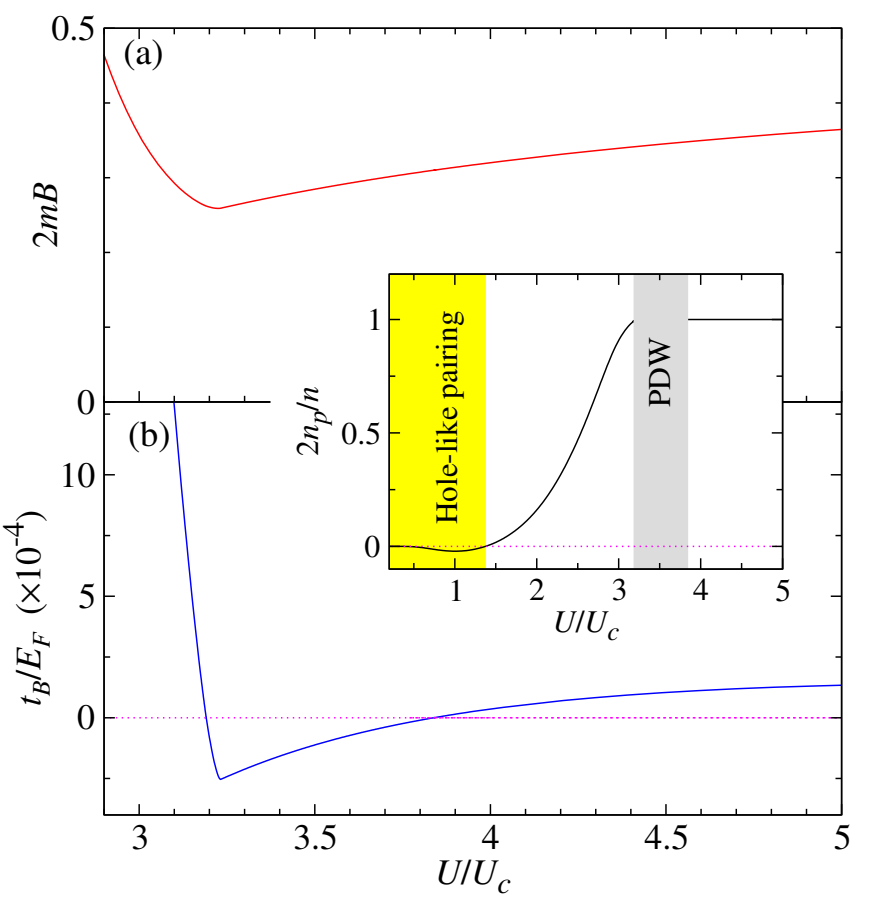

Figure 3. Behavior of (a) $B$ and (b) $t_{B}$ as well as $2 n_{p} / n$ (inset) as a function of $U / U_{c}$, calculated with the same parameters as in Fig. 2 The yellow and grey shaded areas indicate hole-like pairing (with $\left.a_{0}<0\right)$ and PWD state, respectively.

pairing in the $z$-direction always remains particle-like. In the BEC regime, the gap becomes large so that the contributions of the lattice dimensions spread across the entire BZ and become less dominant. As a result, the continuum dimension will dominate the sign of $a_{0}$ in the BEC regime. For hole-like pairing (as shown in the yellow region in the inset of Fig. 3, we have $a_{0}<0$ and thus $n_{p}<0$, as required by the fact $\mu$ is larger than its noninteracting counterpart, via the general relation $n_{p}=n / 2-\sum_{\mathbf{k}} f\left(\xi_{\mathbf{k}}\right)$.

In Fig. 3, we show the corresponding behaviors of (a) the inverse pair mass $B$ and (b) the pairing hopping integral $t_{B}$ as well as pair fraction $2 n_{p} / n$ (inset) as a function of $U / U_{c}$ for $t / E_{F}=0.05$ and $k_{F} d=3$, calculated at $T_{c}$. We set $T=0$ for the PDW states. The behavior of $B$ is rather conventional, with a minimum around $\mu=0$. In contrast, $t_{B}$ is negative in the PDW state, leading to a vanishing $T_{c}$, at a intermediate coupling strength, $3.19 \leq U / U_{c} \leq 3.85$. Upon the sign change of $t_{B}$ from positive to negative, the minimum of $\tilde{\Omega}_{\mathbf{q}}$ will literally move from $\mathbf{q}=0$ to $\mathbf{q}=(\pi / d, \pi / d, 0)$, within the lowest order tight-binding approximation for the in-plane pair dispersion, $\tilde{\Omega}_{\mathbf{q}=\left(q_{x}, q_{y}, 0\right)}$, as shown by the $U / U_{c}=3.5$ case in Fig. 4 (along the $q_{x}$ direction). This corresponds to a first order phase transition from a homogeneous superfluid to a PDW ground state. [27] The physical origin of the PDW states has to do with the strong inter-pair repulsive interaction for relatively large pair size and moderately strong pairing interaction, which leads to Wigner crystallization in the $x y$ plane. It remains unclear and will be left for future studies whether the PDW phase can sustain superfluidity and thus 


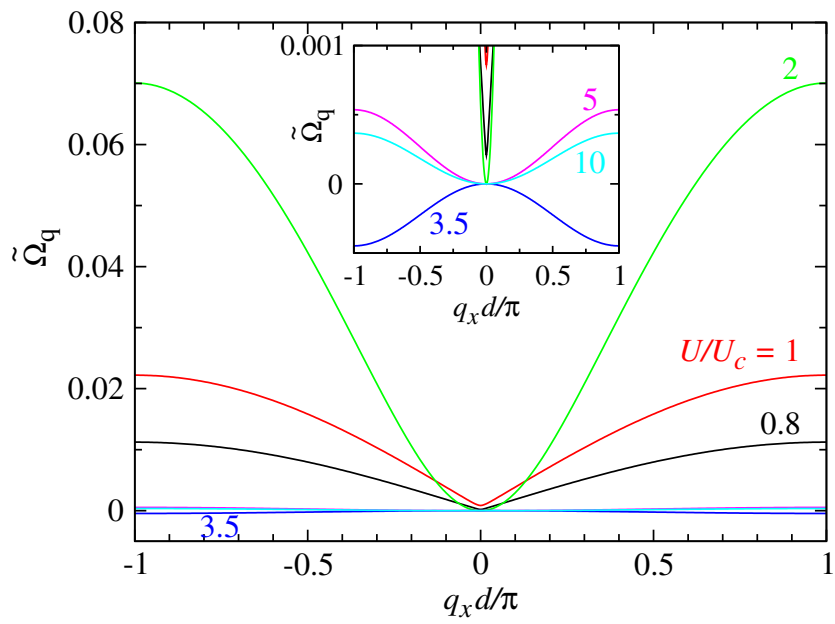

Figure 4. Pair dispersion $\tilde{\Omega}_{\mathbf{q}=\left(q_{x}, 0,0\right)}$ along the $q_{x}$ direction for different $U / U_{c}$, as labeled, calculated with the same parameters as in Fig. 2. For $U / U_{c}=3.5$, the $\Gamma$ point, $\left(q_{x}, q_{y}, q_{z}\right)=0$, becomes a local maximum of $\tilde{\Omega}_{\mathbf{q}=\left(q_{x}, 0,0\right)}$ in the $q_{x}$ and $q_{y}$ directions, signaling an instability of the conventional pair condensation at $q=0$, which hence leads to a PDW state.

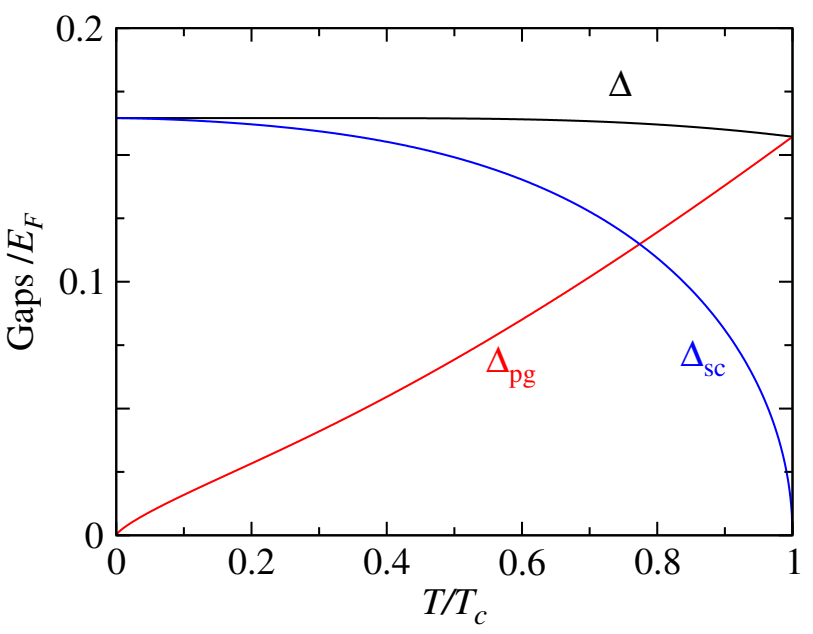

Figure 5. Temperature dependence of the gaps and the order parameter at and below $T_{c}$ at unitarity, $U / U_{c}=1$, for $k_{F} d=2$ and $t / E_{F}=0.05$. The order parameter $\Delta_{s c}$ decreases with $T$ and necessarily closes at $T_{c}$. At very low $T, \Delta_{p g}^{2} \propto n_{\text {pair }} \propto T^{3 / 2}$ so that $\Delta_{p g} \propto T^{3 / 4}$, which increases with $T$. Note that there is already a rather large (pseudo)gap at $T_{c}$ in the unitary regime.

becomes a supersolid. Note that $\tilde{\Omega}_{\mathbf{q}}$ becomes gapped when $a_{0}<0$, which reduces the pseudogap.

Next, we show in Fig. 5 the typical behaviors of the order parameter $\Delta_{\mathrm{sc}}$ and the gaps $\Delta$ and $\Delta_{\mathrm{pg}}$ as a function of temperature at unitarity. They are qualitatively similar to their counterpart in the 3D continuum and 3D lattice cases. As $T$ increases from zero, the order parameter decreases and vanishes at $T_{c}$, while the pseudgap increases from zero and reaches its maximum at $T_{c}$. The increase of $\Delta_{\mathrm{pg}}$ reflects the nature that it results from finite momentum pairing, whose

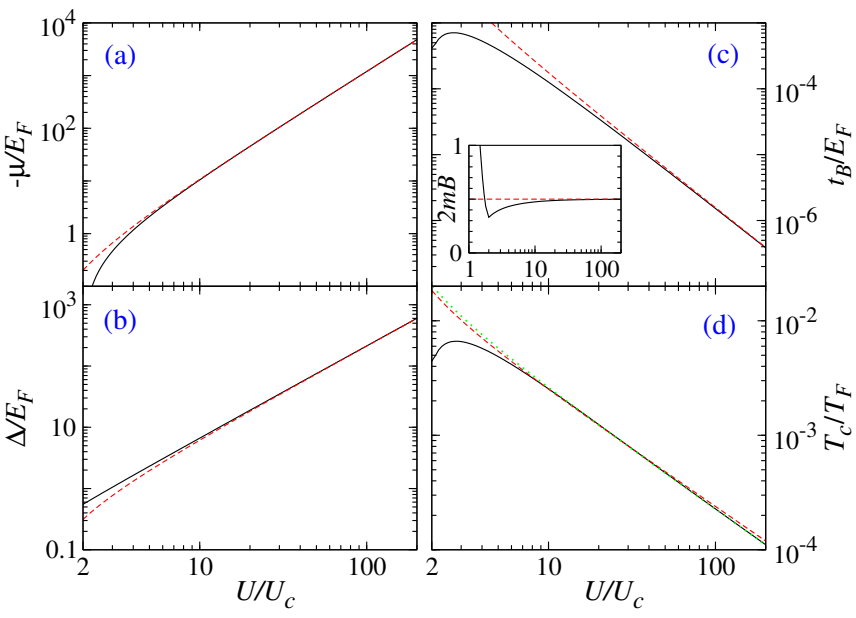

Figure 6. Comparison between full numerical solutions (black solid) and BEC asymptotic behaviors (red dashed lines) of (a) $-\mu$, (b) $\Delta$,(c) $t_{B}$ and $B$ (inset), and (d) $T_{c}$ as a function of $U / U_{c}$ on a log-log scale, for $t / E_{F}=0.05$ and $k_{F} d=2$. The green dotted line contains higher order contributions in the expansion of $L i_{1 / 2}^{-2}(1-z)$

population depends on thermal activation. The combined total gap $\Delta$ follows the BCS mean-field-like behavior, and would continue to exist up to $T^{*}$ far above $T_{c}$. As a function of interaction strength, as $T_{c}$ decreases rapidly with decreasing $U / U_{c}$ towards the BCS regime, all gaps decrease and the ratio $\Delta_{\mathrm{pg}}\left(T_{c}\right) / \Delta(0)$ decreases and becomes essentially zero in the extreme BCS limit. In contrast, with increasing $U / U_{c}$ towards the BEC regime, this ratio approaches unity, while all gaps increase. In this case, $\Delta$ becomes essentially temperature independent, and system can be regarded as a composite Bose gase.

Finally, we show in Fig. 6 the BEC asymptotic behavior (red dashed lines) of (a) $-\mu$, (b) $\Delta$, (c) $t_{B}$ and $B$ (inset) and (d) $T_{c}$ as a function of $U / U_{c}$ on a $\log -\log$ scale for $t / E_{F}=$ 0.05 and $k_{F} d=2$, obtained from Eqs. 4 6 and (8). For comparison, also plotted are the fully numerical results (black solid lines) from Eqs. (1)-(3). It is obvious that our asymptotic solution works well for $U / U_{c} \gtrsim 10$. Importantly, we see that $T_{c}$ is governed by $\sqrt{B t_{B}}$ in the BEC regime, as in Eq. 8. A better asymptotic expression for $T_{c}$, shown as the green dotted line in Fig. 6(d), can be obtained by including higher order effect via fitting $L i_{1 / 2}^{-2}(1-z)$ with a power law in the range of $z \in[0,0.01]$, which yields $T_{c}=2.22\left(n d^{2} \sqrt{B}\right)^{0.964} t_{B}^{0.518}$.

It should be noted that the lattice constant $d$ is governed by the laser wavelength, such as $532 \mathrm{~nm}$ or $1064 \mathrm{~nm}$, which usually cannot be tuned continuously in a typical laboratory. However, one can tune the density $n$ such that the dimensionless parameter $k_{F} d$ can be varied continuously. As for the parameters $t$ and $U$, normally, one can tune the ratio of $U / t$ by changing the lattice depth, as described in Ref. [28]. Since here $t$ is measured in terms of the Fermi energy $E_{F}$, therefore, one can tune $t / E_{F}$ by the density $n$ as well. We note, however, $k_{F}$ and $E_{F}$ are locked together via $E_{F}=\hbar^{2} k_{F}^{2} / 2 m$. Nevertheless, there is already a large room for tuning these 
parameters experimentally. More sophisticated devices, such as a free electron laser [29] or a laser that allows contnuous wavelength tuning [30], may be used to allow independent continuous tuning of $d$. In this case, all parameters can be tuned independently.

\section{CONCULSIONS}

In summary, we study the superfluid transition of a Fermi gas in a 2DOL in the context of BCS-BEC crossover, and pay close attention to the exotic effect of lattice-continuum mixing. We find that, for relatively large $d$ and small $t$ in a 2DOL, a PDW ground state emerges and $T_{c}$ vanishes at intermediate pairing strength. In the BCS and unitary regimes, the nature of the in-plane and overall pairing may change from particlelike to hole-like, with a negative coefficient $a_{0}<0$, and the chemical potential $\mu$ may first increase with increasing pairing strength. In the BEC regime, $T_{c} \approx \frac{\sqrt{6} n t d^{4}}{m}|U|^{-1}$, with $\mu \sim-U^{2}$ and pairing gap $\Delta \sim|\mu|^{3 / 4} \sim|U|^{3 / 2}$. These asymptotic scaling behaviors are qualitatively distinct from their counterpart in the 1DOL, 3D continuum or 3D lattice cases. Our theoretical predictions can be tested in future experiments.

\section{ACKNOWLEDGMENTS}

This work was supported by the NSF of China (Grant No. 11774309 and No. 11674283), and the NSF of Zhejiang Province of China (Grant No. LZ13A040001).
[1] Q. J. Chen, J. Stajic, S. N. Tan, and K. Levin, BCS-BEC crossover: From high temperature superconductors to ultracold superfluids, Phys. Rep. 412, 1 (2005)

[2] I. Bloch, J. Dalibard, and W. Zwerger, Many-body physics with ultracold gases, Rev. Mod. Phys. 80, 885 (2008)

[3] C. Chin, R. Grimm, P. Julienne, and E. Tiesinga, Feshbach resonances in ultracold gases, Rev. Mod. Phys. 82, 1225 (2010)

[4] I. Bloch, Ultracold quantum gases in optical lattices, Nat. Phys. 1, 23 (2005)

[5] M. Köhl, H. Moritz, T. Stöferle, K. Günter, and T. Esslinger, Fermionic atoms in a three dimensional optical lattice: Observing Fermi surfaces, dynamics, and interactions, Phys. Rev. Lett. 94, 080403 (2005)

[6] U. Schneider, L. Hackermüller, S. Will, T. Best, I. Bloch, T. A. Costi, R. W. Helmes, D. Rasch, and A. Rosch, Metallic and insulating phases of repulsively interacting fermions in a $3 \mathrm{D}$ optical lattice, Science 322, 1520 (2008).

[7] P. Nozières and S. Schmitt-Rink, Bose condensation in an attractive fermion gas: from weak to strong coupling superconductivity, J. Low Temp. Phys. 59, 195 (1985)

[8] Q. J. Chen, I. Kosztin, B. Jankó, and K. Levin, Superconducting transitions from the pseudogap state: d-wave symmetry, lattice, and low-dimensional effects, Phys. Rev. B 59, 7083 (1999)

[9] J. B. Wang, L. F. Zhang, Y. Yu, C. H. Lee, and Q. J. Chen, Superfluidity and pairing phenomena in ultracold atomic Fermi gases in one-dimensional optical lattices. I. Balanced case, Phys. Rev. A 101, 053617 (2020)

[10] J. B. Wang, L. F. Zhang, Y. Yu, C. H. Lee, and Q. J. Chen, Superfluidity and pairing phenomena in ultracold atomic Fermi gases in one-dimensional optical lattices. II. Effects of population imbalance, Phys. Rev. A 101, 053618 (2020)

[11] M. M. Parish, S. K. Baur, E. J. Mueller, and D. A. Huse, Quasione-dimensional polarized Fermi superfluids, Phys. Rev. Lett. 99, 250403 (2007)

[12] D. Roscher, J. Braun, and J. E. Drut, Inhomogeneous phases in one-dimensional mass-and spin-imbalanced Fermi gases, Phys. Rev. A 89, 063609 (2014)

[13] E. H. Zhao and W. V. Liu, Theory of quasi-one-dimensional imbalanced Fermi gases, Phys. Rev. A 78, 063605 (2008)

[14] Y.-a. Liao, A. S. C. Rittner, T. Paprotta, W. Li, G. B. Partridge, R. G. Hulet, S. K. Baur, and E. J. Mueller, Spin-imbalance in a one-dimensional Fermi gas, Nature 467, 567 (2010)

[15] G. Pagano, M. Mancini, G. Cappellini, P. Lombardi, F. Schäfer, H. Hu, X.-J. Liu, J. Catani, C. Sias, M. Inguscio, et al., A onedimensional liquid of fermions with tunable spin, Nat. Phys. 10, $198(2014)$

[16] M. C. Revelle, J. A. Fry, B. A. Olsen, and R. G. Hulet, 1D to 3D crossover of a spin-imbalanced Fermi gas, Phys. Rev. Lett. 117, 235301 (2016)

[17] P. Fulde and R. A. Ferrell, Superconductivity in a strong spinexchange field, Phys. Rev. 135, A550 (1964), A. I. Larkin and Y. N. Ovchinnikov, Inhomogeneous state of superconductors, Sov. Phys. JETP 20, 762 (1965), [Zh. Eksp. Teor. Fiz. 47, 1136 (1964)].

[18] G. B. Partridge, W. Li, R. I. Kamar, Y. A. Liao, and R. G. Hulet, Pairing and phase separation in a polarized Fermi gas, Science 311, 503 (2006)

[19] L. F. Zhang, Y. M. Che, J. B. Wang, and Q. J. Chen, Exotic superfluidity and pairing phenomena in atomic Fermi gases in mixed dimensions, Sci. Rep. 7, 12948 (2017)

[20] L. F. Zhang, J. B. Wang, Y. Yu, and Q. J. Chen, Ultra high temperature superfluidity in ultracold atomic Fermi gases with mixed dimensionality, Sci. China - Phys. Mech. Astron. 63, $227421(2020)$

[21] Q. J. Chen, I. Kosztin, B. Jankó, and K. Levin, Pairing fluctuation theory of superconducting properties in underdoped to overdoped cuprates, Phys. Rev. Lett. 81, 4708 (1998)

[22] We also drop the volume $V$, which can be set to unity and will be canceled out in the end.

[23] For 1DOL and 3D continuum, $U$ is renormalized down to zero in the contact limit so that an appropriate parameter is scattering length $a$. We have $\mu \sim-e^{d / a}$ and $\mu \sim-1 / a^{2}$ for the two cases, respectively.

[24] See Eq. (2.4) of Ref. [31].

[25] In principle, $\mu$, when positive, can be measured experimentally by detecting $k_{\mu}=\sqrt{2 m \mu}$ (in the $z$ direction) on the Fermi surface directly via measurement of the fermion momentum distribution, e.g., using the time-of-flight imaging technique.

[26] Y. M. Che, J. B. Wang, and Q. J. Chen, Reentrant superfluidity and pair density wave in single-component dipolar Fermi gases, Phys. Rev. A 93, 063611 (2016) 
[27] When higher order wavevectors are included in the pair dispersion, the minimum may be shifted to a different location.

[28] D. Jaksch, C. Bruder, J. I. Cirac, C. W. Gardiner, and P. Zoller, Cold bosonic atoms in optical lattices, Phys. Rev. Lett. 81, 3108 (1998)

[29] J. Madey, M. O. Scully, and P. Sprangle, The free electron laser: conceptual history, Physica Scripta 91, 083003 (2016)
[30] X. Zhang, A. Liu, C. Lu, and D. Tang, Continuous wavelength tuning in micromachined littrow external-cavity lasers, IEEE Journal of Quantum Electronics 41, 187 (2005)

[31] Q. J. Chen, Generalization of BCS theory to short coherence length superconductors: A BCS-Bose-Einstein crossover scenario, Ph.D. thesis. University of Chicago (2000), available as arXiv:1801.06266. 